\title{
Preliminary on-sky results of the CANARY experiment with an ELT-elongated sodium LGS
}

Lisa Bardou ${ }^{\mathrm{a}}$, Eric Gendron ${ }^{\mathrm{a}}$, Gérard Rousset ${ }^{\mathrm{a}}$, Damien Gratadour ${ }^{\mathrm{a}}$, Alastair G. Basden ${ }^{\mathrm{b}}$, Domenico Bonaccini Calia ${ }^{c}$, Tristan Buey ${ }^{a}$, Mauro Centrone ${ }^{\mathrm{d}}$, Fanny Chemla ${ }^{\mathrm{h}}$, Jean-Luc Gach $^{\mathrm{e}, \mathrm{f}}$, Gianluca Lombardi ${ }^{\mathrm{g}}$, Enrico Marchettic ${ }^{\mathrm{c}}$, Timothy J. Morris ${ }^{\mathrm{b}}$, Richard M. Myers ${ }^{\mathrm{b}}$, James Osborn $^{\mathrm{b}}$, Matthew Townson ${ }^{\mathrm{b}}$, Fabrice Vidal ${ }^{\mathrm{a}}$, and Marcos Reyes García-Talavera ${ }^{\mathrm{i}}$

${ }^{a}$ LESIA, Observatoire de Paris, PSL Research University, CNRS, Sorbonne Universités, UPMC

Univ. Paris 06, Univ. Paris Diderot, Sorbonne Paris Cité, 5 place Jules Janssen, F-92190 Meudon, France

${ }^{b}$ Department of Physics, Durham University, South Road, Durham DH1 3LE, UK

'European Southern Observatory, D-85748 Garching, Germany

dINAF-OAR National Institute for Astrophysics, Via Frascati 33, I-00078 Monte Porzio

Catone (RM), Italy

${ }^{e}$ First Light Imaging S.A.S., Europarc Sainte Victoire Bâtiment 6, Route de Valbrillant, Le Canet, F-13590 Meyreuil, France

${ }^{\mathrm{f}}$ Laboratoire d'Astrophysique de Marseille, 38 rue F. Joliot-Curie, F-13388 Marseille Cedex 13, France

gGran Telescopio Canarias, Cuesta de San José, s/n - E-38712, Breña Baja, La Palma, Spain ${ }^{\mathrm{h}}$ GEPI, Observatoire de Paris, PSL Research University, CNRS, Univ. Paris Diderot, Sorbonne Paris Cité, 5 place Jules Janssen, 92190 Meudon, France

${ }^{\mathrm{i} I n s t i t u t o}$ de Astrofisica de Canarias, Via Lactea, E-38205 La Laguna, Tenerife, Spain

\begin{abstract}
We present on-sky results of wavefront sensing on an elongated LGS. These results are derived from observations made with the multi-object AO demonstrator CANARY on the William Herschel Telescope (WHT) in La Palma. The laser guide star is produced using ESO's transportable Wendelstein LGS unit placed 40 meters away from the WHT so as to replicate the elongations that will be seen on the European ELT.

In this analysis, we evaluate the impact of spot truncation and sub-sampling on the wavefront measurements made with the elongated LGS.

\section{INTRODUCTION}

Laser Guide Stars (LGS) are necessary to take full advantage of the size and field of view of the future Extremely Large Telescopes (ELTs). As such, sodium LGS are included in the design of many wide field Adaptive Optics (AO) systems of the different ELTs : the Giant Magellant Telescope ${ }^{1}$ the Thirty Meter Telescope ${ }^{2}$ and the European Extremely Large Telescope. ${ }^{3,4}$ Unfortunately their use becomes challenging on such large telescopes. The thickness and variability of the mesospheric sodium layer in which the LGS is lighted produce an elongated spot with a varying shape. This complicates wavefront sensing and requires to find new strategies to mitigate the impact of this elongation.

In order to study this problem with realistic data, an on-sky experiment with an elongated LGS has been set up. ${ }^{5}$ It uses the AO demonstrator CANARY, ${ }^{6}$ situated on the William Herschel Telescope (WHT) (pupil diameter of 4.2 meters) in La Palma, along with the European Southern Observatory's (ESO) Wendelstein LGS Unit. ${ }^{7}$ The goals of this experiment are manifold. First, we want to gather data in a variety of conditions with regard to sodium profile, seeing condition and LGS position in the field of view. Then, we aim at deriving an error budget on wavefront sensing with the elongated LGS. Finally, we wish to use these results to compare them with numerical simulations and upgrade the latter to make them closer to reality.
\end{abstract}


In this particular study, SH patterns sampled by the detector are modified to emulate the behavior of different SH configurations. First, by discarding the outer pixels of the sub-aperture, i.e. windowing, sub-apertures with a smaller number of pixels, resulting in a smaller field of view, are simulated. Secondly, by binning the pixels together, pixels with a larger field of view are reproduced. Then the impact of this changes on wavefront sensing performance can be analyzed in order to evaluate which compromises can be made in designing an ELT-scale LGS WFS.

To present those results, we will begin by explaining the set-up of the experiment, with details on AO configuration, data acquisition and observation strategy. We also describe the data processing applied before discussing the outcome of that processing.

\section{EXPERIMENTAL SET-UP}

\subsection{Telescopes}

Let us begin with the largest scale of the experiment with the simultaneous use of several telescopes. The central one is the WHT, on which the AO system operates. From its viewpoint, the LGS must be elongated, therefore the laser launch telescope is placed 40 meters away. Next to the launch telescope is a small telescope (356 $\mathrm{mm}$ ) that monitors the return flux from the LGS. In addition, we use the neighboring Isaac Newton Telescope, of pupil diameter 2.5 meters, situated 400 meters away to image synchronously the sodium profile with high resolution. $^{8}$

\subsection{AO set-up}

If we zoom in on the WHT, we find the AO demonstrator CANARY. First, there is an on-axis LGS WFS looking at the elongated LGS. A reference is then needed to validate the accuracy of the LGS wavefront sensing. Therefore, the LGS is superimposed on a Natural Guide Star (NGS) from the point of view of the WHT. Correspondingly, there is a second on-axis SH wavefront sensors called Truth Sensor(TS) looking at the NGS.

Both on-axis WFS have the same wavefront sampling with 7 by 7 sub-apertures to facilitate the comparison of the measurement coming from each WFS. However the LGS WFS has very large sub-aperture field of view compared to the Truth Sensor in order to accommodate for the size of the elongated LGS. The characteristics of all WFS are summed up in Table 1.

The LGS WFS has some additional characteristics. Its field stop has the size of two sub-apertures in order to be sure not to truncate the elongated spot. There is a narrow bandpass filter around $589 \mathrm{~nm}$ to reject the light from the NGS. It also has a dedicated steering mirror to compensate for laser jitter. Finally, it is motorized so as to be able to conjugate itself to different altitudes. This allows to track the sodium layer as the observed target moves closer or away from zenith and therefore the distance to the sodium layer decrease or increase.

In addition, there are 3 off-axis WFS. They provide additional tomographic data on the turbulence. Their characteristics are also detailed in table 1.

All five WFS see the same 52 actuators Deformable Mirror (DM) as well as a tip-tilt (TT) mirror.

\subsection{Data acquisition}

Once on-sky, we proceed by acquisitions of $10 \mathrm{~s}$, with the system running at $150 \mathrm{~Hz}$, resulting in long data sets. During an acquisition, we register synchronously raw pixels from the cameras, actuators voltage of the mirrors, slopes computed by the real time computer and a sodium profile extracted from the images recorded at the INT. In this paper we work with slopes recomputed off-line from the raw $\mathrm{SH}$ images. 


\begin{tabular}{|c|c|c|c|c|c|c|}
\hline Type of WFS & $\begin{array}{c}\text { Number of } \\
\text { sub-apertures }\end{array}$ & $\begin{array}{c}\text { Pixel field of } \\
\text { view }\end{array}$ & $\begin{array}{l}\text { Sub-aperture } \\
\text { field of view }\end{array}$ & $\begin{array}{l}\text { Number of } \\
\text { pixel per } \\
\text { sub-aperture }\end{array}$ & Camera type & $\begin{array}{c}\text { Read-out } \\
\text { noise }\end{array}$ \\
\hline $\begin{array}{c}\text { on-axis LGS } \\
\text { WFS }\end{array}$ & \multirow{5}{*}{$7 \times 7$} & $0.65^{\prime \prime}$ & $19.5^{\prime \prime}$ & 30 & OCAM $^{2}$ & $\begin{array}{c}<0.2 e^{-} \mathrm{rms} \\
\text { per pixel }\end{array}$ \\
\hline $\begin{array}{c}\text { on-axis NGS } \\
\text { WFS (TS) }\end{array}$ & & $0.23^{\prime \prime}$ & $3.7^{\prime \prime}$ & \multirow{4}{*}{16} & \multirow{4}{*}{ ANDOR } & \multirow{4}{*}{$\begin{array}{l}<0.6 e^{-} \mathrm{rm} \\
\text { per pixel }\end{array}$} \\
\hline $\begin{array}{c}\text { off-axis NGS } \\
\text { WFS } \# 1\end{array}$ & & $0.28^{\prime \prime}$ & $4.48^{\prime \prime}$ & & & \\
\hline $\begin{array}{c}\text { off-axis NGS } \\
\text { WFS } \# 2\end{array}$ & & $0.26^{\prime \prime}$ & $4.16^{\prime \prime}$ & & & \\
\hline $\begin{array}{c}\text { off-axis NGS } \\
\text { WFS } \# 3\end{array}$ & & $0.29^{\prime \prime}$ & $4.64^{\prime \prime}$ & & & \\
\hline
\end{tabular}

TABLE 1: Canary WFS characteristics.

\subsection{Observing strategies}

Since there are 3 off-axis NGS WFS, all of our observational targets are asterisms comprising four NGS of magnitude 8 to 11 (in $\mathrm{R}$ band). They are contained in a field of view of $2.5^{\prime}$.

We first proceed to take measurements without the laser. They provide a lower limit of the difference between measurements coming from the two on-axis WFS. The results from this calibration are presented in section 4.1.

The loop has been closed on the LGS WFS, ${ }^{9}$ but in this study we focus on open loop data. Depending on the acquisitions, the steering mirror can be locked on the LGS WFS.

While in open loop, data is regularly acquired while dithering the tip-tilt mirror. From these acquisitions is derived an estimation of the centroid gain, which is then used to correct the measured slopes by.

The data from all NGS WFS are also used in longer acquisitions of 10000 frames. With these data sets, a tomographic reconstruction of the turbulence is performed, which can be used to estimate the impact of cone effect.

\subsection{Observations}

So far, the experiment has benefited from 3 runs of observations. The first one, of four nights, took place at the end on July 2016. A second run of 5 nights occurred during September 2016. All the data that we are going to discuss comes from the September run. Another run of four nights happened at the beginning of June 2017, and a last one is scheduled for end of September, beginning of October 2017 and will last 5 nights.

Further details on the experiment can be found in Reeves et al. ${ }^{10}$ in this conference.

\section{DATA REDUCTION}

\subsection{Pipeline}

The wavefront reconstruction is performed with Zernike polynomials. Before reconstruction, the slopes obtained from centroiding on the SH images are corrected by the centroid gain. Working with Zernike polynomials allows discarding tip-tilt from the analysis and treating separately the focus and higher orders. The higher orders span Zernike polynomials 5 to 36 as labeled by Noll. Once the Zernike reconstruction has been computed for each of the two on-axis wavefront sensors, we work on the difference between the two wavefronts. Here we concentrate on the temporal variance of the higher orders of this wavefront difference, that is to say, we analyze only the dynamical aspect of the problem. Finally, we evaluate the noise contribution to the variance in order to subtract it from the total variance. The noise is first evaluated from the slopes corrected by the centroid gain, then it is projected on the Zernike modes to allow subtraction from the total wavefront difference variance. For the sake of clarity, the final results are given in $\mathrm{nm}$ rms and so are actually the standard deviations. 


\subsection{Slope computation}

The slopes are computed from SH patterns with algorithms based on brightest pixels selection followed by center of gravity computation. The Truth Sensor is always centroided with 50 brightest pixels. As for the LGS WFS, we begin by spanning a large range of brightest pixels. Then we artificially change the design of the WFS by applying binning or windowing on the SH images. The binning of pixels emulates pixels with a bigger on-sky field of view while the windowing emulates sub-apertures with a smaller field of view. Exploring those parameters gives information on which compromise can be reached in the design of the LGS WFS for the ELT.

\section{RESULTS}

\subsection{Minimum wavefront difference between the on-axis WFS}

As said before, the first step is the calibration of the minimum wavefront difference between the TS and the LGS WFS. This is obtained with on-sky measurements without the laser. Both WFS then observe the central star of the asterism targeted. The acquisitions used were made while observing two different asterisms named A53 and A349.

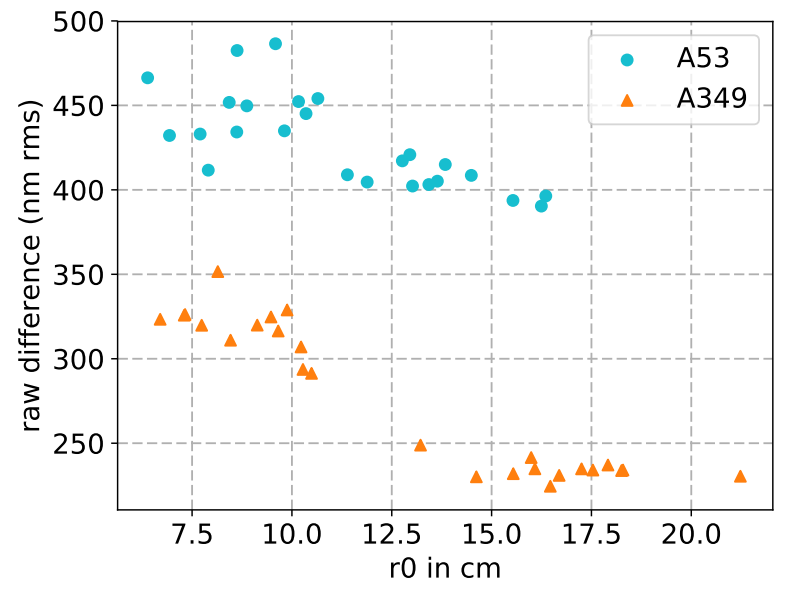

(a)

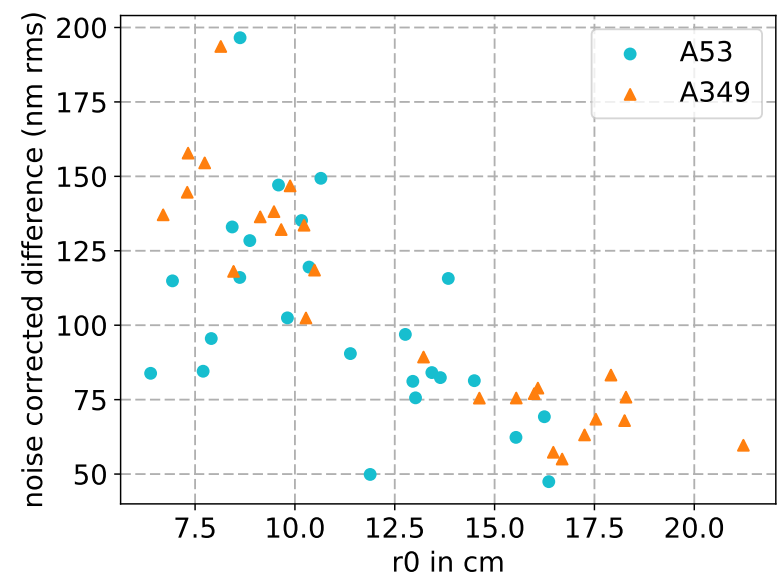

(b)

FiguRE 1: In these two figures, each point represents an acquisition of 10s. Temporal variance of the wavefront difference between the TS and the LGS WFS are plotted against $r_{0}$. The $r_{0}$ are computed by fitting the distribution predicted by Noll $^{11}$ according to the strength of the turbulence to the variance of the different Zernike coefficients. On the left figure, the variance of the wavefront difference is computed on the higher orders as defined in section 3.1. On the right, the noise contribution to the variance has been removed from the data presented on the left.

This analysis is shown in Figure 1. In Figure 1a we see two distinct levels in the plot. They correspond to the two different targets and can be explained by a fainter central star for A53, thus producing more noisy measurement. And indeed, once the noise variance is subtracted, in Figure 1b, there is no longer two separated levels. At lower $r_{0}$, the wavefront differences measured have higher values which is consistent with a stronger turbulence. There are also large variations from one measurement to the other which is consistent with the noisier measurements produced by a stronger turbulence. For higher $r_{0}$, both the values and dispersion of the measurements are lower. The conclusion from this calibration is that the lower wavefront difference achievable is of roughly $100 \mathrm{~nm}$ for strong turbulence, and $50 \mathrm{~nm}$ for weaker turbulence.

\subsection{Data selection}

During the run of September we observed four different targets. The data presented here comes from observations of all four targets. The characteristics of each data are presented in Table 2. On Figure 2 is shown an average image of a sub-aperture for each target. 


\begin{tabular}{|c|c|c|c|c|}
\hline Target name & Number of total acquisitions & $\begin{array}{l}\text { Number of acquisitions with } \\
\text { TT mirror modulation }\end{array}$ & Date (UTC) & $r_{0}$ \\
\hline A34 & 26 & 9 & $\begin{array}{l}17^{\text {th }} \text { September : } 22 \mathrm{~h} 27 \text { to } \\
22 \mathrm{~h} 41\end{array}$ & $14 \mathrm{~cm}$ \\
\hline A 53 & 30 & 10 & $\begin{array}{l}18^{\text {th }} \text { September }: 3 \mathrm{~h} 41 \text { to } \\
3 \mathrm{~h} 57\end{array}$ & $13 \mathrm{~cm}$ \\
\hline A349 & 13 & 2 & $\begin{array}{l}19^{t h} \text { September }: 5 \mathrm{~h} 29 \text { to } \\
5 \mathrm{~h} 41\end{array}$ & $7 \mathrm{~cm}$ \\
\hline AT2 & 11 & 4 & $\begin{array}{l}20^{\text {th }} \text { September : } 4 \text { h26 to } \\
4 \mathrm{~h} 32\end{array}$ & $13 \mathrm{~cm}$ \\
\hline
\end{tabular}

TABLE 2: Overwiew of the acquisitions used in the following results. The $r_{0}$ are an average over the different acquisitions.

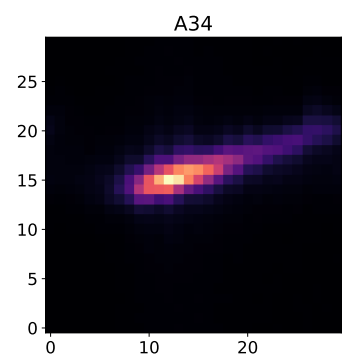

(a)

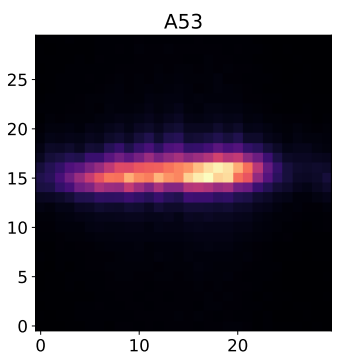

(b)

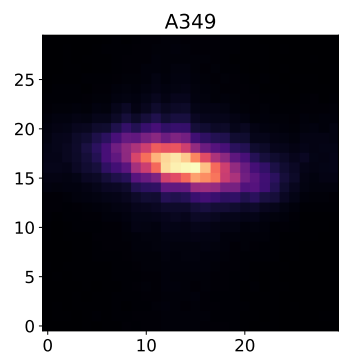

(c)

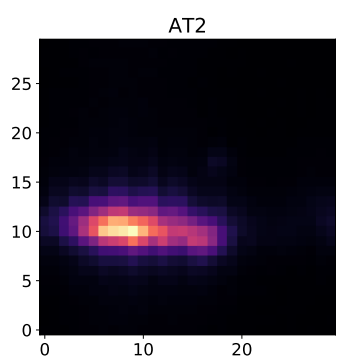

(d)

FiguRE 2: Average of one subaperture over 200 frames for each of the target observed. No recentering has been applied on the LGS spots.

In the subsequent plots, for each SH configuration explored, each target has only one data point : it is the mean obtained with all the acquisitions on that target processed in that configuration. The error bars come from the standard deviation over the acquisitions.

\subsection{Discussion on results}

In the following figures $(3,4,5$ and 6$)$, the centroid gains are shown in Figures labeled (a). The computed slopes are corrected by these centroid gains before the wavefront reconstruction is performed. Then, in Figures labeled (b), the LGS WFS noise measurements are presented. Lastly, the square root of the variance of the difference between the wavefronts measured with the TS and the LGS WFS is presented in Figures (c). In these last Figures, the tip, tilt and focus have been discarded and the noise contribution has been removed. A better result is defined as a lower difference between the wavefronts, because it implies a greater accuracy in the LGS WFS measurement. The standard deviations are given in $\mathrm{nm} \mathrm{rms}$. The centroid gain ideal value is 1 , in which case the measurement agrees with the interaction matrix and pixel scale measured during calibration of the bench.

For the sake of convenience, in the following discussion, we assimilate the name of the asterism targeted (for example A53) with the LGS spot shape observed at that time.

\subsubsection{Brightest pixels}

In Figure 3 we see that the variation of the number of brightest pixels has only a small impact on the final result. Globally, the more brightest pixels there are, the less noise, but the centroid gains drop as the number of brightest pixels increase, so that a compromise has to be found.

If we take a closer look at Figure 3c, the number of brightest pixels has the least influence on A349. It is probably due to the fact that the spot (as seen on Figure 2c) looks mostly symmetric. On A53, the more pixels, the better, which is also coherent with the fact that it presents the largest spot (Figure 2b). AT2 has a minimum : 


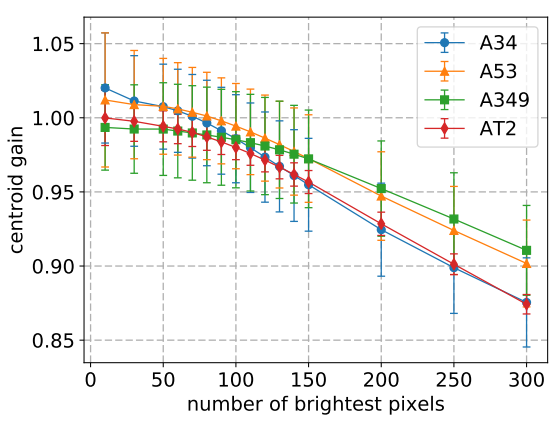

(a)

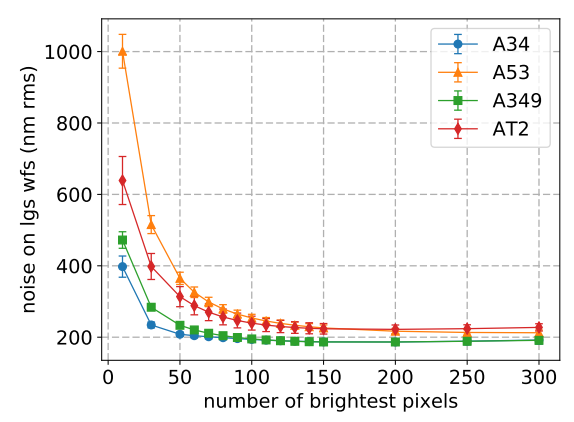

(b)

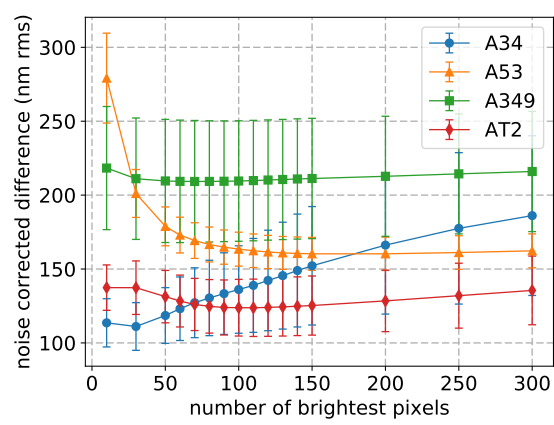

(c)

FigURE 3: Exploration of the influence of the number of brightest pixels

it may be that since the spot present a slight asymmetry (one side is brighter than the other) but is still smaller than A53, at first taking into account more pixels helps to have the whole profile then only noise is added to the center of gravity. At last, for A34 it is better to take as little brightest pixels as possible, above a certain limit below which results are meaningless. This would indicate that it is better to only take the bright 'head' of the spot. It seems to contradict the results from AT2 where the spot also clearly features a brighter part, but the tail of A34 being much fainter may be the cause of this discrepancy.

For the exploration of other algorithms we choose a baseline of 90 brightest pixels as being a good compromise with the different targets.

\subsubsection{Windowing}

Now we explore the impact of diminishing the field of view of a sub-aperture. When the window in which the pixels are selected is narrowed, the number of brightest pixels is also lowered. As one pixel is removed in the size of the window, 3 brightest pixels are removed from the center of gravity.

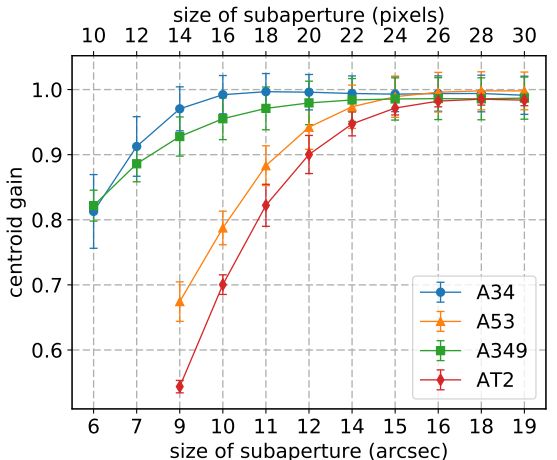

(a)

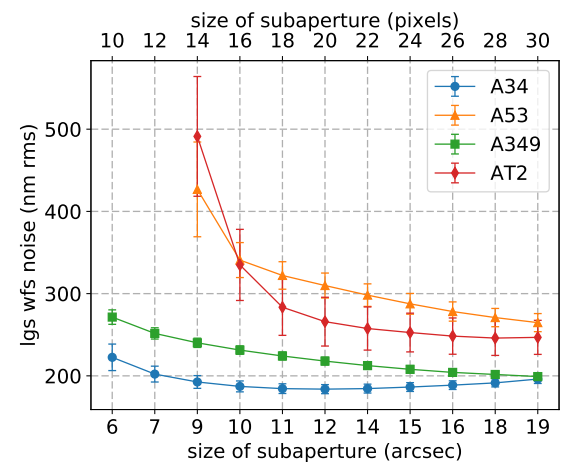

(b)

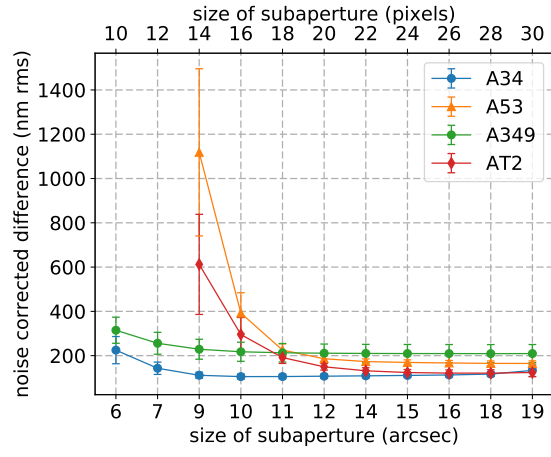

(c)

FIGURE 4: Exploration of the influence of smaller sub-aperture field of view. On the lower abscissa is the total field of view of one sub-aperture and on the upper abscissa is the corresponding number of pixels along one axis of the sub-aperture. Some points have been removed for targets A53 and AT2 : the noise introduced by the truncation makes them irrelevant.

The results are visible in Figure 4. As expected, globally the centroid gain drops and the noise and the wavefront difference rise as the field of view is reduced. But these tendencies become obvious under a $11^{\prime \prime}$ field of view. This can be explained by looking at the spots on figure 2. For A349, to begin with, no significant pixels are lost until the subaperture is left with less than 22 pixels $\left(14^{\prime \prime}\right)$. Then, once even more pixels are discarded, 
we find the same behavior as with a low number of brightest pixels, which, as seen in the previous section, has little impact on the wavefront difference. The same is true with A34: it was shown before that it is better to keep only the bright head in the middle, so a lot of the field of view can be cropped out before having a negative impact on the measurement. The same should be expected with AT2. It is not the case because the spot is not well centered, as can be seen on Figure 2d. Indeed, the actual truncation of the spot begins as soon as 3 pixels are eliminated on the left side of the sub-aperture. With A53, the truncation of the spots begins quickly because of its large spot size. In these last two cases it is noticeable that once the spot is really truncated, the difference between the truth sensor and LGS WFS almost immediately rises. In the end, the actual truncation that can be reached is up to $2^{\prime \prime}$ before the centroid gain loss becomes unacceptable.

Nevertheless, it should be noted that the number of brightest pixels has been fixed for each window size without seeking the optimum. Results can probably be slightly improved by increasing the number of brightest pixels.

\subsubsection{Binning}

In this part binning has been applied to the image, prior to centroiding. 2 by 2 and 3 by 3 binning has been tested. In the first case 22 brightest pixels, and in the second case 10 brightest pixels are selected before applying the center of gravity.

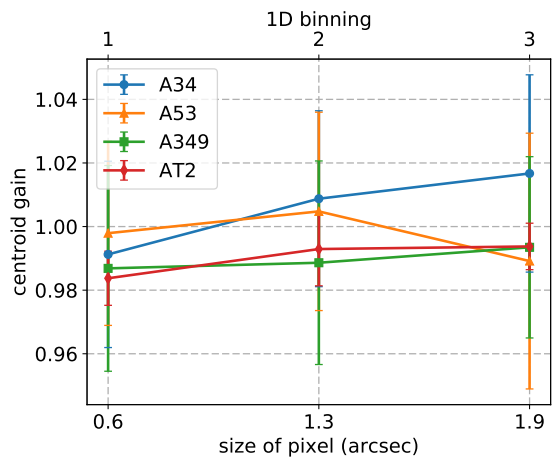

(a)

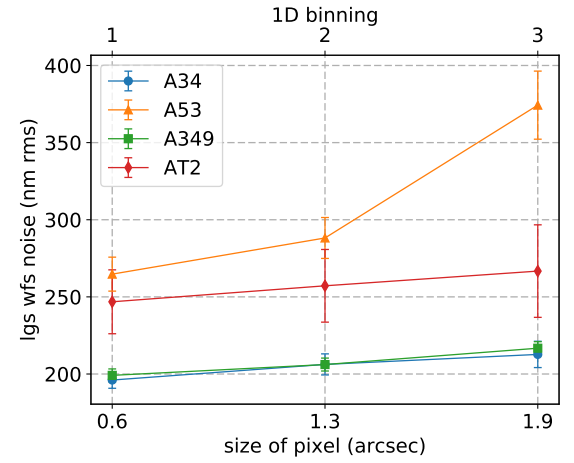

(b)

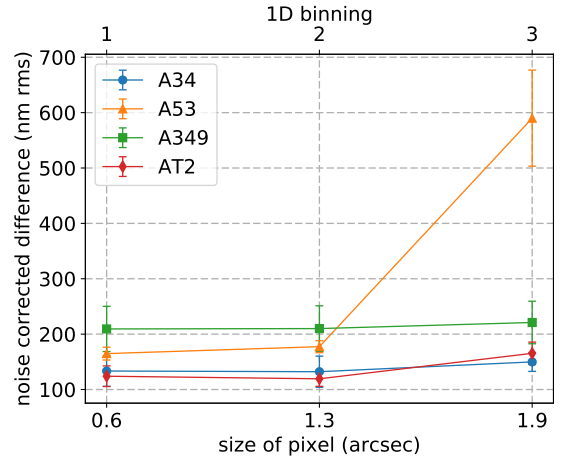

(c)

Figure 5: Exploration of the influence of larger pixel field of view.

The results of this examination are shown in Figure 5. Predictably, the wavefront difference rises as pixels grow bigger, but the increase is very small compared to the changes observed when changing the field of view of the sub-aperture. The larger impact is with 3 by 3 binning on A53, which can be explained by the fact that this spot is almost horizontal, and on its non-elongated axis, the spot is only 3 pixels wide, so when these pixels are binned together, a lot of resolution is lost on this axis.

\subsubsection{Binning and windowing}

Given the little impact of binning on the LGS WFS, we also try windowing after 2 by 2 binning. The corresponding plot are presented in Figure 6.

The same behavior is found as in section 4.3.2. This corroborates the findings in the two previous sections : it is possible to use $1.3^{\prime \prime}$ pixels and $11^{\prime \prime}$ sub-apertures without much increasing the difference between the TS and the LGS WFS measurements.

\section{CONCLUSION}

Preliminary results are presented, in which the wavefronts from two on-axis WFS are compared : an elongated LGS WFS and a NGS WFS 


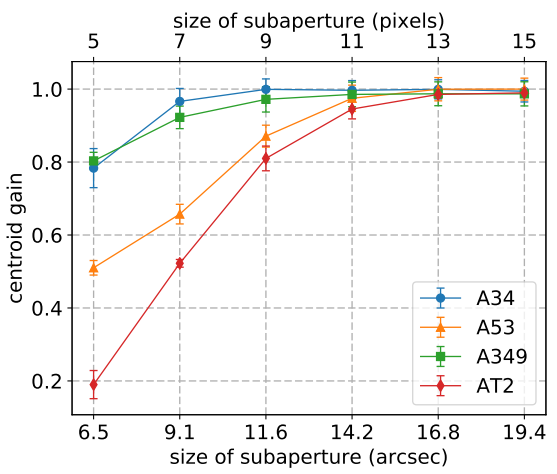

(a)

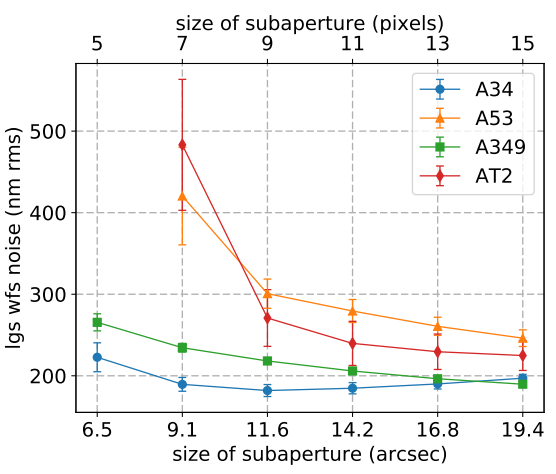

(b)

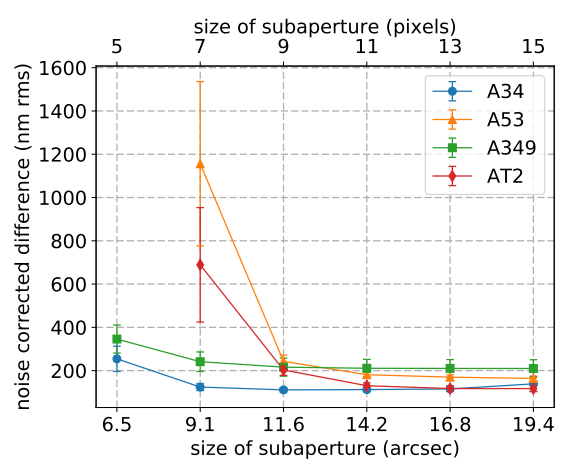

(c)

Figure 6: Applying windowing on a binned image. As in Figure 4, the more meaningless point have been removed to allow better readability.

Wavefront differences between the two on-axis WFS as low as $120 \mathrm{~nm}$ rms are achieved, with a floor difference without LGS of $50 \mathrm{~nm}$ between these two WFS. We have seen that the measurement is not very sensitive to the pixel field of view and pixels of $1.3^{\prime \prime}$ yield accurate measurement. However, according the LGS shape, shrinking the field of view of the sub-aperture can have a large impact for a field of view lower than $11^{\prime \prime}$.

There is much to do to further this study. The first step is to test centroiding based on correlation on the LGS wavefront sensing. This should allow to have results independent of the LGS shape and reach a lower wavefront error. Then we want to advance toward an error budget by estimating the cone effect contribution thanks to the tomographic data of the off-axis WFS. We will also investigate noise models with projections along the elongated axis of the spot. Furthermore, static terms must also be considered to derive a comprehensive wavefront sensing strategy.

Finally, the end goal remains : including the previous results in end-to-end simulations to attain full ELT-scale simulations.

\section{ACKNOWLEDGMENTS}

This work is supported by the OPTICON project (EC FP7 grant agreement 312430) and by the European Southern Observatory. My PhD thesis is funded by the Fondation CFM pour la recherche.

\section{REFERENCES}

[1] Bouchez, A. H., Acton, D. S., Biasi, R., et al., "The Giant Magellan Telescope adaptive optics program," in [Adaptive Optics Systems IV], Proc. SPIE 9148, 91480W (2014).

[2] Boyer, C., Adkins, S., Andersen, D. R., et al., "Adaptive optics program at TMT," in [Adaptive Optics Systems IV], Proc. SPIE 9148, 91480X (2014).

[3] Neichel, B., Fusco, T., Sauvage, J.-F., et al., "The adaptive optics modes for HARMONI : from Classical to Laser Assisted Tomographic AO," in [Adaptive Optics Systems V], Proc. SPIE 9909, 990909 (2016).

[4] Morris, T., Basden, A., Buey, T., et al., "Adaptive optics for MOSAIC : design and performance of the wide(st)-field AO system for the E-ELT," in [Adaptive Optics Systems V], Proc. SPIE 9909, 99091I (2016).

[5] Rousset, G., Gratadour, D., Gendron, E., et al., "Proposal for a field experiment of elongated Na LGS wave-front sensing in the perspective of the E-ELT," in [Adaptive Optics Systems IV], Proc. SPIE 9148, 91483M (2014).

[6] Gendron, E., Vidal, F., Brangier, M., et al., "MOAO first on-sky demonstration with CANARY," A $3 A$ 529, L2 (2011).

[7] Bonaccini Calia, D., Friedenauer, A., Protopopov, V., et al., "PM fiber lasers at 589nm : a 20W transportable laser system for LGS return flux studies," in [Adaptive Optics Systems II], Proc. SPIE 7736, 77361U (2010). 
[8] Townson, M. J., Osborn, J.,; Bardou, L., et al., "Sodium layer statistics from the canary high-resolution sodium profiler," in [Adaptive optics for Extremely Large Telescopes], (2017).

[9] Basden, A. G., Bardou, L. and, B. D., Buey, T., et al., "On-sky demonstration of matched filters for wavefront measurements using elt-scale elongated laser guide stars," Mon. Not. R. Astron. Soc. (2017).

[10] Reeves, A. P., Bardou, L., Basden, A. G., et al., "Canary phase d : On sky testing of the european elt lgs ao configuration," in [Adaptive optics for Extremely Large Telescopes], (2017).

[11] Noll, R. J., "Zernike polynomials and atmospheric turbulence," Journal of the Optical Society of America (1917-1983) 66, 207-211 (1976). 